\title{
Improved orbital solution and masses for the very low-mass multiple system LHS 1070^
}

\author{
A. Seifahrt ${ }^{1,2}$, T. Röll ${ }^{2}$, R. Neuhäuser ${ }^{2}$, A. Reiners ${ }^{1}$, F. Kerber $^{3}$, H. U. Käuff ${ }^{3}$, R. Siebenmorgen ${ }^{3}$, and A. Smette ${ }^{4}$ \\ 1 Universität Göttingen, Institut für Astrophysik, Friedrich-Hund-Platz 1, 37077 Göttingen, Germany \\ e-mail: seifahrt@astro.physik.uni-goettingen.de \\ 2 Universität Jena, Astrophysikalisches Institut und Universitäts-Sternwarte, Schillergässchen 2, 07745 Jena, Germany \\ 3 ESO, Karl-Schwarzschild-Str. 2, 85748 Garching, Germany \\ ${ }^{4}$ ESO, Alonso de Córdova 3107, Vitacura, Casilla 19001, Santiago 19, Chile \\ Received 19 October 2007 / Accepted 16 February 2008
}

\section{ABSTRACT}

\begin{abstract}
We present a refined orbital solution for the components $\mathrm{A}, \mathrm{B}$, and $\mathrm{C}$ of the nearby late-M type multiple system LHS 1070. By combining astrometric datapoints from NACO/VLT, CIAO/SUBARU, and PUEO/CFHT, as well as a radial velocity measurement from the newly commissioned near infrared high-resolution spectrograph CRIRES/VLT, we achieve a very precise orbital solution for the $\mathrm{B}$ and $\mathrm{C}$ components and a first realistic constraint on the much longer orbit of the A-BC system. Both orbits appear to be co-planar. Masses for the $\mathrm{B}$ and $\mathrm{C}$ components calculated from the new orbital solution $\left(M_{\mathrm{B}+\mathrm{C}}=0.157 \pm 0.009 M_{\odot}\right)$ are in excellent agreement with theoretical models, but do not match empirical mass-luminosity tracks. The preliminary orbit of the A-BC system reveals no mass excess for the A component, giving no indication for a previously proposed fourth (D) component in LHS 1070 .
\end{abstract}

Key words. astrometry - stars: binaries - close, stars: low-mass, brown dwarfs

\section{Introduction}

LHS 1070 (GJ 2005, LP 881-64) is a low-mass triple system at a distance of $7.72 \pm 0.15 \mathrm{pc}$ from the Sun (Costa et al. 2005). It consists of two components with masses close to the substellar limit (LHS 1070 B and C) with spectral types of M 8.5 to M 9, orbited by a slightly more massive M 5.5 type component, LHS 1070 A.

This system is important when defining the empirical massluminosity relationship at the low-mass end of the main sequence. Few reliable and accurate mass determinations, such as dynamical masses from binary and multiple systems, exist below $0.1 M_{\odot}$, which makes LHS 1070 an important anchor point, especially given its age of several Gigayears and its proximity to the Sun.

Leinert et al. (2001) have presented the first orbital solution for the LHS 1070 BC system based on speckle measurements and early adaptive optics images. Deriving a combined mass of $0.138 M_{\odot}$ for LHS $1070 \mathrm{BC}$, they put the system in the context of the empirical and theoretical mass-luminosity relationships presented in Leinert et al. (2000). Given the much longer orbital period of the outer, more massive component LHS $1070 \mathrm{~A}$, mass estimates for LHS $1070 \mathrm{~A}$ and a first reliable solution for the outer orbit were beyond reach in 2001. Nevertheless, Leinert et al. (2001) succeeded in giving rough constraints on

\footnotetext{
* Based on observations made with ESO Telescopes at the Paranal Observatory under programme ID 60.A-9078(A) and 79.C-0106(A), as well as data collected at Subaru Telescope, which is operated by the National Astronomical Observatory of Japan.

Based also on observations obtained at the Canada-France-Hawaii Telescope (CFHT) which is operated by the National Research Council of Canada, the Institut National des Sciences de l'Univers of the Centre National de la Recherche Scientifique of France, and the University of Hawaii.
}

the outer orbit, suggesting co-planarity with the inner orbit of LHS 1070 BC.

Late-type double and multiple systems are also important probes for the connection of rotation and magnetic activity at the transition from partly to fully convective stars. During the science verification phase of CRIRES at the VLT (Käufl et al. 2006), we obtained spatially resolved measurements of the rotational velocity and magnetic activity for all three components of LHS 1070 (see Reiners et al. 2007, for details). This measurement is essentially free of the uncertainties induced from the otherwise unknown inclination angle when comparing rotation and magnetic activity for objects of different spectral types, since the rotation axes of the components in this triple system are most likely spatially well-aligned. The CRIRES/VLT datapoint also adds important information on the orbit of the triple system, removing the ambiguity in the true spatial orientation.

In this paper we present our results from an improved orbital fit of LHS 1070 BC and first constraints on the orbit of LHS 1070 A around the barycentre of the BC subsystem. Our observations, as well as extensive archival data, are presented in Sect. 2. The orbital solutions and masses derived from these data are discussed in Sect. 3. A summary of the most important results is given in Sect. 4.

\section{Observations and data reduction}

In addition to the astrometric datapoints published in Leinert et al. (2001), we used archived images from the adaptive optics (AO) cameras PUEO/CFHT and NACO/VLT covering a timespan of nearly 6 years, see Table 1 for details. We also used the first spatially resolved, high-resolution spectrum of LHS 1070 ABC (Reiners et al. 2007) and determined radial velocities (RV) for the relative motions of LHS $1070 \mathrm{~B}$ and C, as well as for the motion of LHS 1070 A around the barycentre of 
Table 1. Observation $\log$ and obtained relative positions for LHS1070 C $\left(X_{\mathrm{C}}, Y_{\mathrm{C}}\right)$ with respect to component $\mathrm{B}$ and for the barycentre of LHS $1070 \mathrm{BC}\left(X_{\mathrm{BC}}, Y_{\mathrm{BC}}\right)$ with respect to component A.

\begin{tabular}{|c|c|c|c|c|c|c|c|c|c|}
\hline Date & Instrument & $\begin{array}{l}\text { Pixel scale } \\
\text { (mas/pixel) }\end{array}$ & Filter & Prog. ID. & PI & $\begin{array}{c}X_{\mathrm{C}}{ }^{a} \\
\text { (mas) }\end{array}$ & $\begin{array}{c}Y_{\mathrm{C}} \\
\text { (mas) }\end{array}$ & $\begin{array}{l}X_{\mathrm{BC}}^{b} \\
\text { (mas) }\end{array}$ & $\begin{array}{c}Y_{\mathrm{BC}} \\
\text { (mas) }\end{array}$ \\
\hline \multicolumn{10}{|c|}{ Observations retrieved from archives } \\
\hline $2000-08-16$ & PUEO/CFHT & 34.9 & $K^{\prime}$ & 00AD99 & Forveille & 25.0 & 229.1 & 1520 & 206 \\
\hline $2000-12-12$ & PUEO/CFHT & 34.9 & $K$ & 00BH12 & Roddier & -24.6 & 218.5 & 1526 & 224 \\
\hline $2002-07-24$ & PUEO/CFHT & 34.9 & $K^{\prime}$ & $02 \mathrm{AF} 43$ & Beuzit & -265.2 & 126.7 & 1541 & 315 \\
\hline $2002-09-11$ & PUEO/CFHT & 34.9 & $H, K^{\prime}$ & $02 \mathrm{BF} 27$ & Beuzit & -281.8 & 116.9 & 1535 & 326 \\
\hline $2002-10-21$ & NACO/VLT & 13.24 & $J, K_{\mathrm{s}}, \mathrm{NB} 2.17$ & 70.C-0476(A) & Bailer-Jones & -295.8 & 109.3 & 1537 & 334 \\
\hline $2002-11-15$ & NACO/VLT & 13.24 & NB2.17 & 70.C-0476(A) & Bailer-Jones & -303.3 & 103.9 & 1540 & 337 \\
\hline $2002-12-16$ & NACO/VLT & 13.24 & $J, K_{\mathrm{s}}$ & 70.C-0704(A) & Leinert & -314.3 & 97.3 & 1538 & 342 \\
\hline $2003-01-25$ & NACO/VLT & 13.24 & $J, K_{\mathrm{s}}, \mathrm{NB} 2.17$ & 70.C-0476(A) & Bailer-Jones & -327.1 & 89.2 & 1536 & 344 \\
\hline $2003-05-24$ & NACO/VLT & 13.24 & $J, K_{\mathrm{s}}, \mathrm{NB} 2.17$ & 70.C-0476(A) & Bailer-Jones & -362.4 & 59.0 & 1534 & 369 \\
\hline 2003-06-11 & NACO/VLT & 13.24 & $K_{\mathrm{s}}$ & 71.C-0262(A) & Bailer-Jones & -365.9 & 55.6 & 1533 & 370 \\
\hline 2003-06-17 & NACO/VLT & 13.24 & $J, K_{\mathrm{s}}, \mathrm{NB} 2.17$ & 70.C-0476(A) & Bailer-Jones & -367.2 & 54.4 & 1534 & 372 \\
\hline 2003-06-27 & NACO/VLT & 13.24 & $J, K_{\mathrm{s}}, \mathrm{NB} 2.17$ & 70.C-0476(A) & Bailer-Jones & -369.8 & 52.5 & 1534 & 375 \\
\hline 2003-09-05 & NACO/VLT & 13.24 & $K_{\mathrm{s}}$ & 71.C-0262(B) & Bailer-Jones & -386.0 & 37.3 & 1533 & 378 \\
\hline 2003-09-14 & NACO/VLT & 13.24 & $J, K_{\mathrm{s}}, \mathrm{NB} 2.17$ & 70.C-0476(A) & Bailer-Jones & -388.3 & 35.1 & 1533 & 381 \\
\hline $2003-12-10$ & NACO/VLT & 13.24 & NB1.64 & 72.C-0570(A) & Beuzit & -405.8 & 14.8 & 1532 & 390 \\
\hline $2003-12-12$ & NACO/VLT & 13.24 & $J, H, K_{\mathrm{s}}$ & 72.C-0022(A) & Leinert & -406.3 & 14.8 & 1531 & 391 \\
\hline 2004-12-11 & NACO/VLT & 13.24 & $K_{\mathrm{s}}$ & 74.C-0637(A) & Köhler & -443.6 & -73.3 & 1511 & 446 \\
\hline 2006-10-30 & NACO/VLT & 13.24 & NB2.17 & 78.C-0386(A) & Ratzka & -347.2 & -197.1 & 1458 & 524 \\
\hline \multicolumn{10}{|c|}{ Own observations } \\
\hline 2006-10-09 & CRIRES/VLT & & & 60.A-9078(A) & Reiners & & & & \\
\hline $2007-07-16$ & NACO/VLT & 13.24 & NB & 79.C-0106(A) & Neuhäuser & -260.7 & -223.7 & 1426 & 563 \\
\hline $2007-07-25$ & CIAO/SUBARU & 10.82 & NB & o07111 & Neuhäuser & -258.2 & -224.4 & 1431 & 567 \\
\hline
\end{tabular}

${ }^{a} X_{\mathrm{C}}$ and $Y_{\mathrm{C}}$ are accurate to \pm 1.5 mas. ${ }^{b} X_{\mathrm{BC}}$ and $Y_{\mathrm{BC}}$ are accurate to \pm 6 mas.

LHS 1070 BC. Finally, in July 2007 we observed LHS 1070 in two imaging campaigns with NACO/VLT and CIAO/SUBARU.

All AO imaging data were reduced by combining individual exposures (shift and add) and determining the positions and fluxes of each object (including their errors) with IDL/starfinder (Diolaiti et al. 2000), a program based on empirical PSF fitting. In cases where a high number of frames were recorded, the errors were calculated from statistics over the distribution of the single positions obtained from the individual frames instead of the formal error computations on a single co-added frame.

The pixel scale and detector orientation of PUEO/CFHT were determined from reference images of the HIPPARCOS double star HIP482 ( $\left.\rho=7.687^{\prime \prime}, \theta=148.29^{\circ}\right)$ obtained during each of the respective runs. The pixel scale was stable at $34.9 \pm$ $0.1 \mathrm{mas} /$ pixel, but the detector orientation was changing between the runs by up to $\pm 2.5^{\circ}$, and was stable during each of the runs on the $0.05^{\circ}$ level. For all NACO/VLT observations taken with the $\mathrm{S} 13$ camera, a fixed pixel scale of $13.24 \pm 0.05$ mas/pixel, and a detector orientation of $\theta=0.0 \pm 0.1^{\circ}$ was adopted. Our experience from other long-term monitoring programmes (see i.e. Neuhäuser et al. 2007) show that both, the pixel scale and the detector orientation of NACO/VLT are very stable, and the accuracy of their absolute value is limited by the astrometric errors of available HIPPARCOS double stars rather than instrumental instabilities. Finally, our CIAO/SUBARU images were calibrated by comparing an HST/WFPC image of the core region of the globular cluster M 15 with images of the very same region in M 15 taken during our run with CIAO/SUBARU.

We note that for the NACO/VLT observations the dominant error source is the absolute pixel scale and detector orientation rather than the centroiding error on the targets. The latter can easily be below 1 mas, especially when enough frames with good $\mathrm{S} / \mathrm{N}$ were obtained. However, the typical accuracy of NACOs S13 pixel scale is about \pm 0.05 mas/pix, which in turn limits the accuracy of the determined positions to about \pm 1.5 mas for the typical separation of LHS1070 BC $\left(\sim 0.5^{\prime \prime}\right)$ and \pm 6 mas for the much larger separation of LHS1070 BC around component A. Datapoints being closer in time than a few days were combined to a mean epoch since the expected motions are far smaller than the derived errors.

The data reduction and calibration of our CRIRES/VLT spectrum is outlined in Reiners et al. (2007). Since here we derive relative radial velocities, our result critically depends on a good dispersion solution. The covered spectral range falls into a clear atmospheric window. CRIRES is equipped with a Th-Ar hollow cathode lamp for wavelength calibration (Kerber et al. 2007). More than 2000 lines have been established as wavelength calibration standards by laboratory measurements in a collaboration between the US National Institute of Standards and Technology (NIST) and ESO (Kerber et al. in prep.). These calibration reference data are implemented in the CRIRES data reduction package. We made use of this linelist to calibrate our data.

For the respective relative radial velocity between each of the components of LHS1070, the alignment of the components in the slit is crucial. At the time of the observation, all three components are nearly, but not perfectly, aligned along the slit. The misalignment (as a deviation from a perfect linear configuration) was $\sim 9^{\circ}$. From geometrical considerations (and by taking the individual $\mathrm{S} / \mathrm{N}$ ratios into account), we adopt $\pm 1.5 \mathrm{~km} \mathrm{~s}^{-1}$ for the uncertainty in the relative RV between LHS 1070 B and C, and for the relative RV between LHS $1070 \mathrm{~A}$ and the barycentre of LHS1070 BC. We note that the most important constraint for the orbital solution is given by the sign of the radial velocity rather than by its exact value. 
Table 2. Orbital and system parameters for LHS 1070 B and C.

\begin{tabular}{lcl}
\hline \hline Parameter & Value & Error \\
\hline$a\left(^{\prime \prime}\right)$ & 0.4619 & \pm 0.0007 \\
$e$ & 0.034 & \pm 0.002 \\
$i\left(^{\circ}\right)$ & 62.59 & \pm 0.12 \\
$\Omega\left({ }^{\circ}\right)$ & 14.50 & \pm 0.12 \\
$\omega\left(^{\circ}\right)$ & 240.33 & \pm 0.24 \\
$P($ days $)$ & 6214.74 & \pm 0.42 \\
$T_{0}(\mathrm{MJD})$ & 48305.6 & \pm 1.8 \\
& & \\
$\pi_{\text {trig }}\left({ }^{\prime \prime}\right)$ & $129.47^{a}$ & \pm 2.48 \\
$d(\mathrm{pc})$ & $7.72^{a}$ & \pm 0.15 \\
$a(\mathrm{AU})$ & 3.57 & \pm 0.07 \\
$M_{\mathrm{B}}+M_{\mathrm{C}}\left(M_{\odot}\right)$ & 0.157 & $\pm 0.009^{b}$ \\
\hline
\end{tabular}

${ }^{a}$ From Costa et al. (2005). ${ }^{b}$ Including error in distance, at fixed distance the error is $0.0007 M_{\odot}$.

\section{Analysis and results}

\subsection{Astrometric solution and orbital fitting: LHS 1070 BC}

To fit the relative orbit of LHS1070 B and C around their common barycentre, we chose a similar approach to the one presented in Leinert et al. (2001). We simultaneously solved the Keplerian equation using an IDL routine based on Hilditch (2001) for all astrometric and spectroscopic datapoints. A $\chi^{2}$ minimisation was performed to find the optimal set of parameters, and a Jacknife approach (Shao \& Dongsheng 1996) was chosen to determine the errors in the orbital parameters (see Table 2 for the parameters and Fig. 1 for the orbital fit). The orbital coverage is now $253^{\circ}$. The reduced $\chi^{2} \simeq 1.27$ is reasonable but points towards a slight underestimation of the real uncertainties in the original dataset of Leinert et al. (2001) and in the NACO/VLT data.

We note that the refined orbital parameters of the BC system agree with the ones published in Leinert et al. (2001), but only the time of periastron $\left(T_{0}\right)$ differs slightly, being 1991-02-18 in our calculation. The error on the orbital period and semimajor axis is now at the sub-percent level, allowing a very precise mass determination for a fixed-distance estimate to the system.

\subsection{Astrometric solution and orbital fitting: LHS 1070 A}

The datapoints for the orbit of the BC system around LHS1070 A presented in Leinert et al. (2001) did not show any strong curvature, hence, the authors used a general $\chi^{2}$ fit with combinations of period and eccentricity to map the parameter space. They used a stability argument for hierarchical triple systems to further constrain the period and inclination angle. The new datapoints reveal a strong curvature, the ascending node being probably close in time to our CRIRES/VLT radial velocity datapoint. Thus, the new datapoints give a stronger constraint on the outer orbit, event hough the covered arc of the orbit is still only about $33^{\circ}$. However, the orbital solution is still not unique. We performed the $\chi^{2}$ minimisation for fixed pairs of eccentricity and orbital period, spanning a grid of $e=0 \ldots 0.45$ and $P=40 \ldots 120 \mathrm{yr}$. Three families of solutions appeared to fit the data: first a small region where the eccentricity is zero and the orbital period is $\sim 82$ years, and second, two narrow regions for rising eccentricity with either shorter or longer periods with the longitude of periastron $(\omega)$ turning by $180^{\circ}$ from $\sim 150^{\circ}$ to $\sim 327^{\circ}$ between these two solutions (see Fig. 3 ).
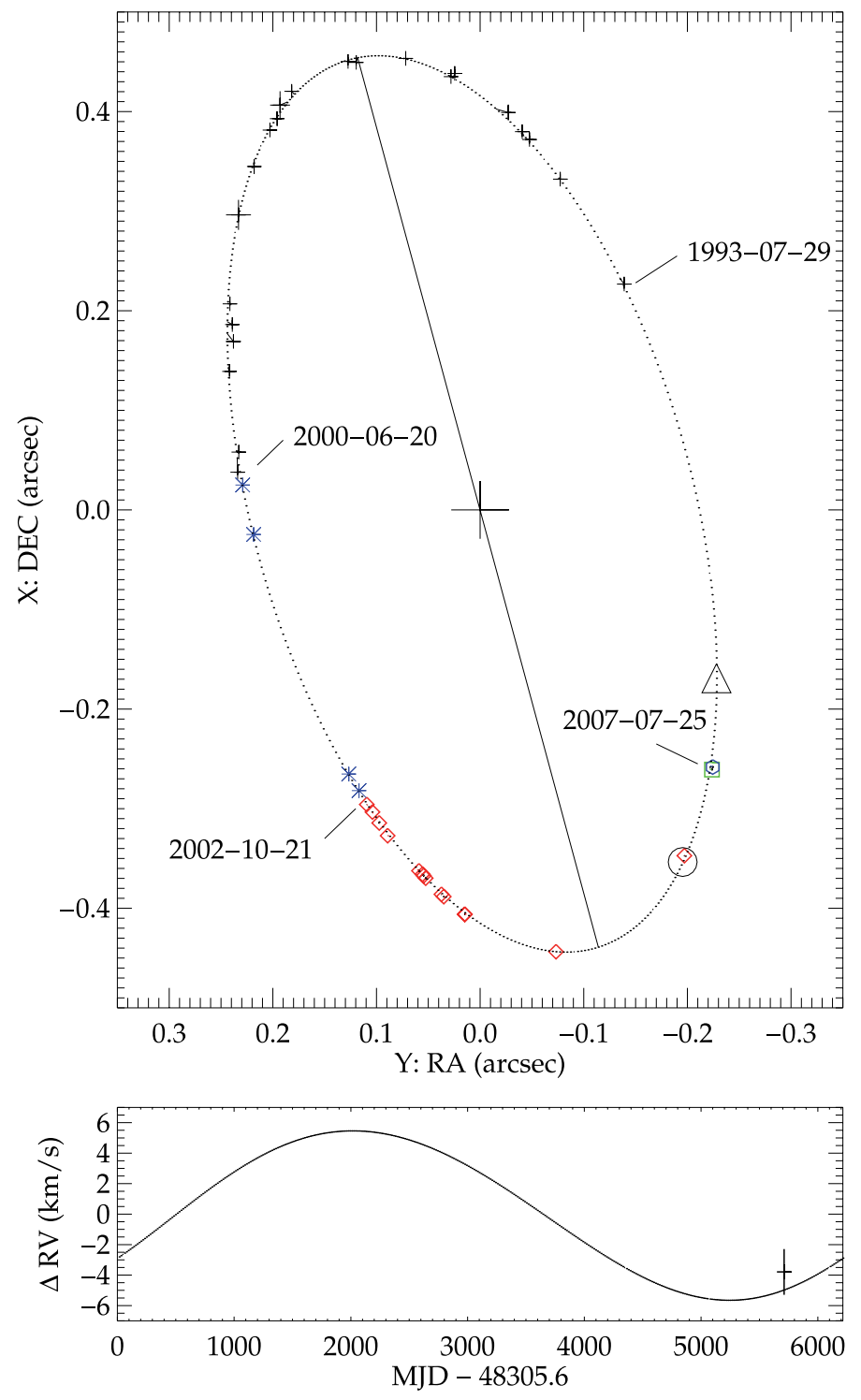

Fig. 1. Relative orbit of LHS $1070 \mathrm{C}$ around component B. Upper panel: astrometric observations from Leinert et al. (2001) (black crosses) including error bars, PUEO/CFHT archival datapoints (blue asterisks), NACO/VLT archival datapoint (red diamonds), along with a new NACO/VLT datapoint (green box) and a new CIAO/SUBARU datapoint (blue hexagon). Errors and derivations $(\mathrm{O}-\mathrm{C})$ for all datapoints except the original points from Leinert et al. (2001) are smaller than their respective symbols. The time of the radial velocity measurement (200610-09) is marked with a circle, the periastron with a triangle. The line of nodes is shown. Lower panel: relative radial velocity between component $\mathrm{C}$ and $\mathrm{B}$. The CRIRES/VLT datapoint is shown with its $1 \sigma$ error bar.

We have to use a stability criterion to further constrain the parameter space, as in Leinert et al. (2001). Here we use the critical distance parameter $q_{2} / a_{1}$ from Harrington (1975), where $q_{2}=a_{2}\left(1-e_{2}\right)$, with updated values from Donnison \& Mikulskis (1995) to infer the stability limit of the LHS 1070 system. Given a prograde orbital motion, the mass estimates of the three bodies and solutions for the semimajor axis of the BC system $\left(a_{1}\right)$, as well as the values for the eccentricity $\left(e_{2}\right)$ and semimajor axis $\left(a_{2}\right)$ of the wide orbit from our grid solution, we can plot 

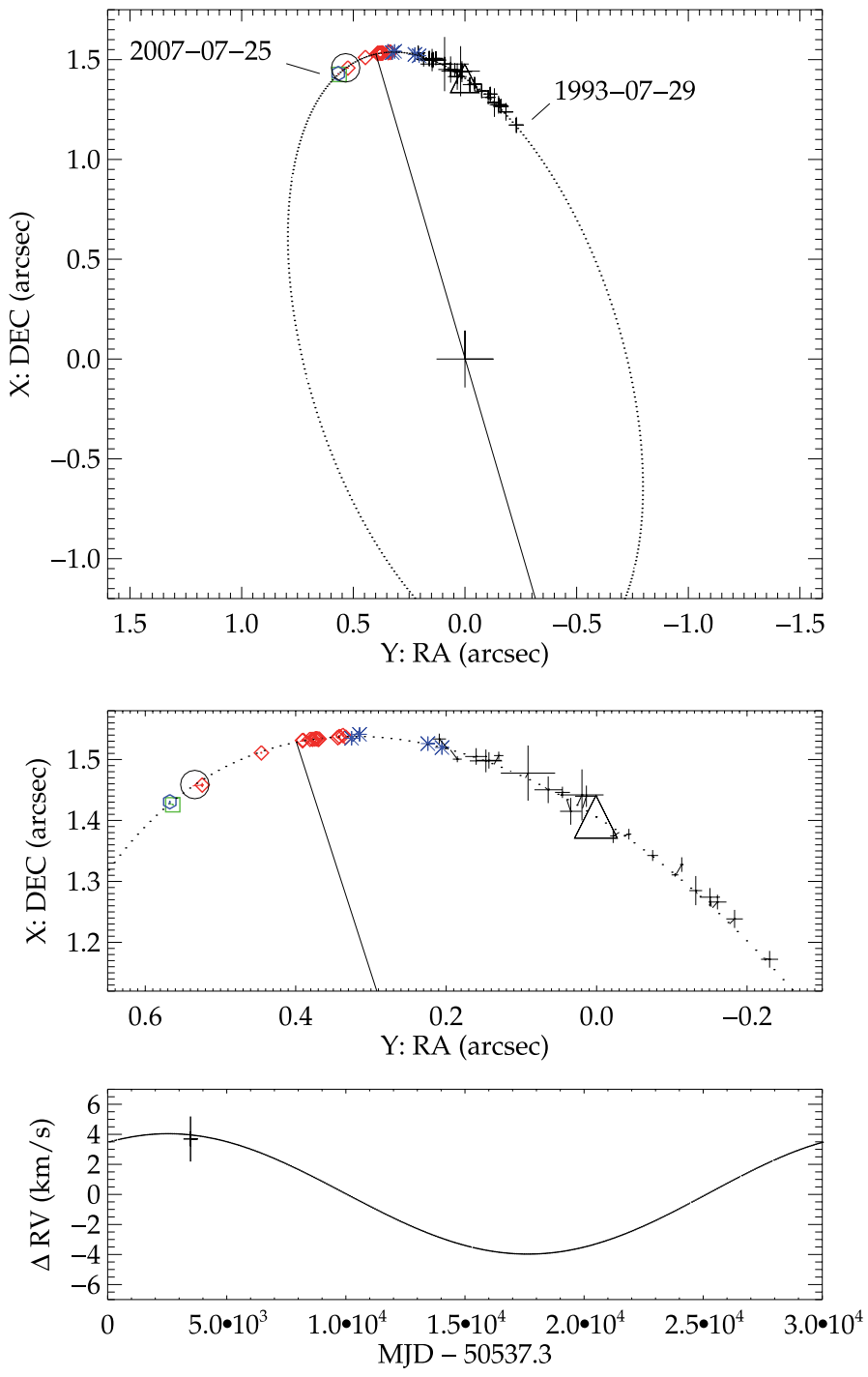

Fig. 2. Relative orbit of the barycentre of LHS 1070 B and C around component A. Upper panel: astrometric observations. Same notation and symbols as in Fig. 1. Central panel: cutout of the upper panel for better visibility. Lowest panel: relative radial velocity between the barycentre of LHS $1070 \mathrm{~B}$ and C and component A. The CRIRES/VLT datapoint is shown with its $1 \sigma$ error bar.

the line of stability ${ }^{1}\left(q_{2} / a_{1} \geq 3.3\right)$ in the $P-e$ plane of Fig. 3 . Only solutions below and to the right of this line are long-term stable, excluding most of the regions with orbital periods shorter than 80 years and eccentricities greater than zero. This is similar to what Leinert et al. (2001) propose, based on slightly different assumptions.

Having narrowed down the phase-space of possible solutions for the outer orbit considerably, we evaluate the histograms of the most important orbital parameters for solutions within $2 \sigma$ around $\min \left(\chi^{2}\right)$ and within the stability region (see Fig. 4). We find that the distribution of the inclination angle for the outer orbit is within $2^{\circ}$ of the value found for the BC orbit $\left(\sim 62.6^{\circ}\right)$ and $\Omega$ is nearly identical for both orbits $\left(\sim 14^{\circ}\right)$. Hence, the inner and outer orbits appear to be co-planar. In Fig. 2 we show the datapoints and the most likely orbital solution with $P \simeq 82 \mathrm{yr}$ and $e=0.01$.

\footnotetext{
${ }^{1}$ Stability is here defined as no significant change in the orbital parameters over a significant number of orbital revolutions.
}

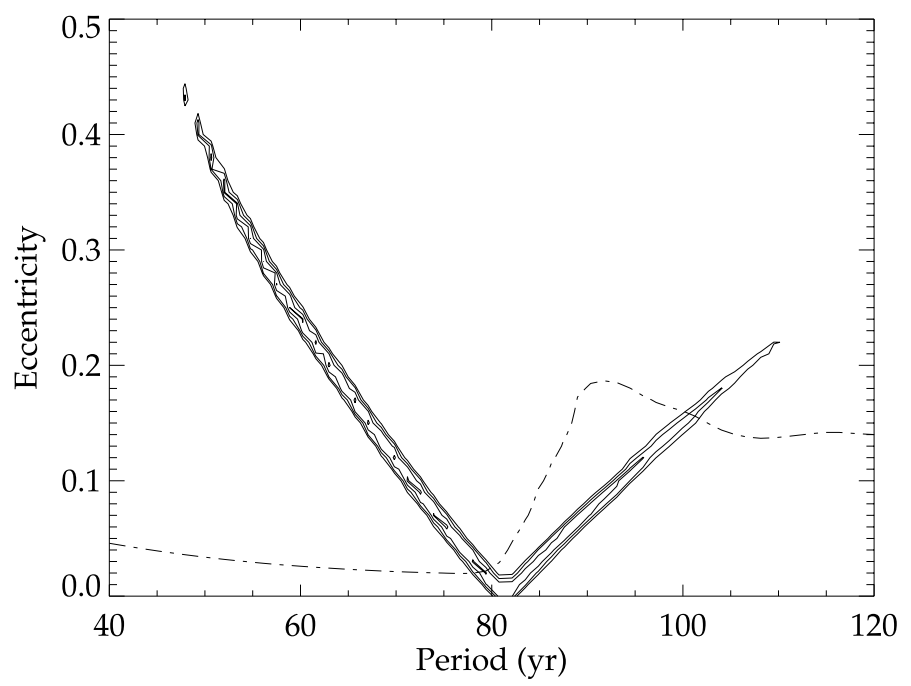

Fig. 3. The most likely values of eccentricity and orbital period for the outer orbit (barycentre of LHS $1070 \mathrm{BC}$ around component A). The contours show the 1,2 , and $3 \sigma$ contours around the $\chi^{2}$ minimum. The left branch represents a family of solutions where $\omega \simeq 150^{\circ}$, while the right branch is for a family of solutions where $\omega \simeq 327^{\circ}$. The dashdotted line marks the stability threshold of the system (see text for details). Only solutions below this line are long-term stable.

\subsection{Masses}

With the new orbit and refined distance (Costa et al. 2005) of the LHS1070 system, we can constrain the combined dynamical mass of the $\mathrm{B}$ and $\mathrm{C}$ components to $M_{\mathrm{B}}+M_{\mathrm{C}}=0.157 \pm 0.009 M_{\odot}$. The dominating error source for the dynamical mass of LHS $1070 \mathrm{BC}$ is still the uncertainty of $\pm 0.15 \mathrm{pc}$ in the distance of the system. At a fixed distance of $7.72 \mathrm{pc}$, the error in mass is as small as $0.0007 M_{\odot}$. Our value for the combined mass of LHS $1070 \mathrm{BC}$ is higher by $9 \%$ than the one obtained by Leinert et al. (2001), mainly because the new distance estimate by Costa et al. (2005) is higher by nearly $3 \%$ than the value adopted by Leinert et al. (2001). This improvement moves the datapoints for LHS $1070 \mathrm{~B}$ and C closer to the $5 \mathrm{Gyr}$ isochrone in Leinert et al. (2001, Fig. 5); see also Fig. 5 here. The combined dynamical mass of LHS1070 B and C is thus in very good agreement with the model predictions for the combined mass of LHS1070 B and $\mathrm{C}$ of $0.159 \ldots 0.161 M_{\odot}$ from Leinert et al. (2000) (based on Baraffe et al. 1998). Hence, both components are slightly above the hydrogen-burning mass limit.

We note that the mass predictions from empirical massluminosity relations differs from our value by a considerable amount. Leinert et al. (2000) give a combined mass for LHS1070 B and $\mathrm{C}$ of $\simeq 0.138 M_{\odot}$ from optical, and $\simeq 0.115 \ldots 0.130 M_{\odot}$ from the near infrared mass-luminosity relations based on Henry et al. (1999) and Henry \& McCarthy (1993). Such predictions underestimate the true mass by 12 to $26 \%$.

The first constraint on the orbit of LHS1070 A around components $\mathrm{BC}$ allows the calculation of a combined mass for the whole system. The error budget for the combined mass of LHS1070 ABC is still dominated by the uncertainties in the parameters of the outer orbit (semimajor axis and orbital period). However, orbital period and semimajor axis are strongly correlated, and their respective error contributions do not simply add up. The combined dynamical mass of the whole system of LHS $1070 \mathrm{ABC}$ is $M_{\mathrm{LHS} 1070}=0.272 \pm 0.017 M_{\odot}$. 

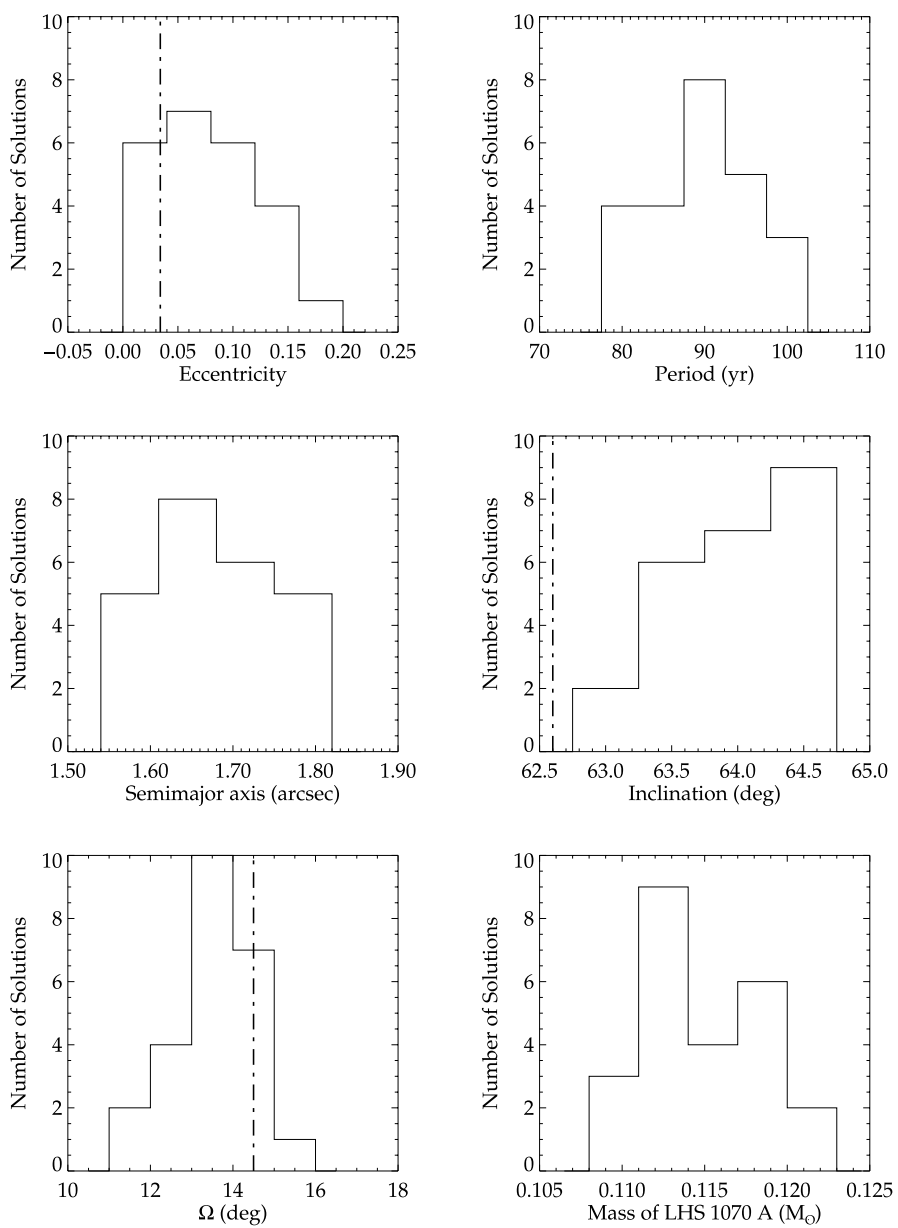

Fig. 4. Histograms of the distribution of orbital parameters from our grid of possible solutions for the outer orbit within $2 \sigma$ around $\min \left(\chi^{2}\right)$ and within the stability region inferred for the outer orbit. The mass of LHS 1070 A was derived from the combined system mass and the mass of LHS 1070 BC (see Table 1). The spread in mass does not include errors from the distance of the system. The dashed-dotted lines indicate the respective values from the inner orbit (LHS 1070 BC) for comparison.

Subtracting the well-constrained dynamical mass of LHS1070 BC from the total dynamical mass of the system leaves $M_{A}=0.115 \pm 0.010 M_{\odot}$ for the A component. The precision is higher than for the total mass estimate of LHS $1070 \mathrm{ABC}$ since the error contribution from the uncertainty in distance nearly cancels out. The resulting dynamical mass for LHS1070 A is only slightly higher than the predictions, both from theoretical models of $0.098 \ldots 0.109 M_{\odot}$ and the empirical tracks of $0.097 \ldots 0.113 M_{\odot}$ (Leinert et al. 2000; Baraffe et al. 2003).

In Fig. 5 we show the mass-luminosity relation with datapoints from Delfosse et al. (2000) and Henry et al. (1999). LHS $1070 \mathrm{ABC}$ is shown in this graph with absolute $V$ magnitudes obtained from Leinert et al. (2000) and our new mass estimates. Note that the error margin for the mass of LHS $1070 \mathrm{~B}$ and $\mathrm{C}$ are shown twice as large to accommodate the uncertainty in the mass ratio of both components. As becomes apparent from this plot, the empirical mass-luminosity relation is too flat, underpredicting masses below $0.1 M_{\odot}$, while the model prediction from Baraffe et al. (1998) is much steeper and fits the available datapoints.

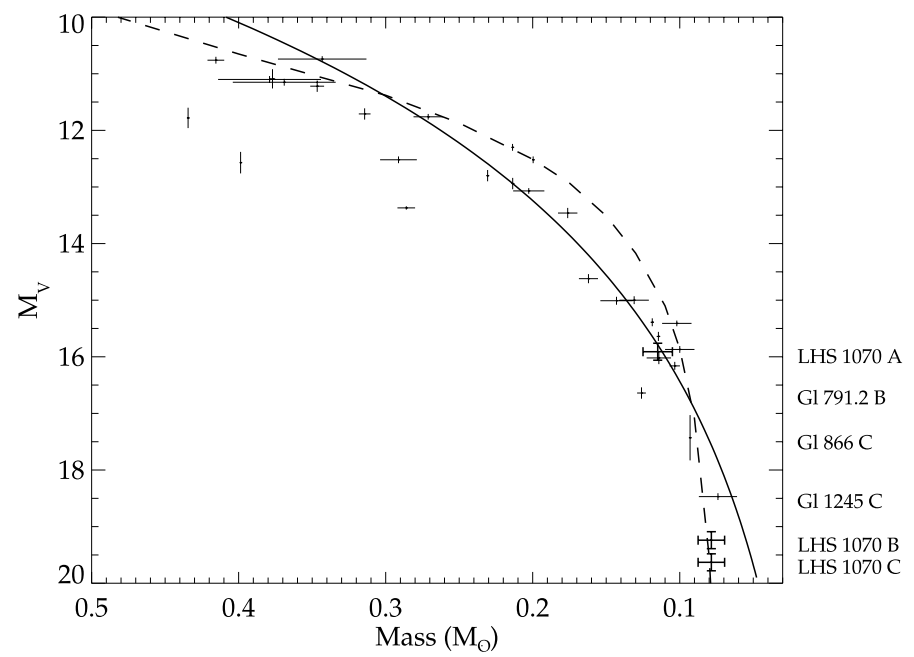

Fig. 5. Mass-luminosity relationship at the end of the main sequence. Data taken from Delfosse et al. (2000), except for GJ 1245 C (Henry et al. 1999) and LHS $1070 \mathrm{ABC}$ (this paper, $M_{V}$ from Leinert et al. 2000). The solid line shows the empirical mass-luminosity relationship from Henry et al. (1999, Eq. (7)) and the dashed line the theoretical relationship from Baraffe et al. (1998).

\subsection{Is there a fourth component in the system?}

Henry et al. (1999) report the discovery of a fourth component in the LHS 1070 system from HST FGS measurements. The authors give a magnitude difference of $\Delta V \simeq 2.5$ to LHS $1070 \mathrm{~A}$, a mass of $0.07 M_{\odot}$ for LHS $1070 \mathrm{D}$, and a separation of $\sim 50$ mas from LHS1070 A at their HST measurements. Leinert et al. (2000) find no clear presence of such a rather massive, but still much cooler and unresolved component in the spectrum of LHS1070 A.

We checked all images, especially the ones observed with NACO/VLT under good atmospheric conditions (hence with a good strehl ratio) for any additional object close to LHS $1070 \mathrm{~A}$. We did not find any object at distances equal to or greater than the diffraction limit of the VLT at $J, H$, and $K_{\mathrm{s}}$ band (32 to 56 mas, respectively). We did not find any indication of an elongation or any difference in the PSF shape of LHS 1070 A, B, and $\mathrm{C}$. This rules out the existence of a component with more than $0.060 M_{\odot}$ at distances over 32 mas of $\Delta J \leq 4.5 \mathrm{mag}$ and at distances over 56 mas of $\Delta K_{\mathrm{s}} \leq 5.0 \mathrm{mag}$, based on Baraffe et al. (2003) and our detection limits. Given the extended time coverage and tight sampling of the NACO/VLT datapoints, it is rather unlikely that we have always missed the object because the distance of both components was too small to be resolved.

Leinert et al. (2001) argue that the reflex motion of an object of intermediate mass between LHS1070 A and D should induce a reflex motion of the order of 10 mas, which was not detectable with their dataset. Analysing the residuals of the later NACO/VLT measurements, we indeed find a scatter of 1-3 mas, exhibiting a non-Gaussian distribution. However, given the large separation of the observations in comparison to the presumed orbital period of the tentative fourth component, we are unable to find an orbital solution for these points. We can only argue that the detected derivations are considerably smaller than the prediction of Leinert et al. (2001), implying a much lower mass than previously anticipated.

Our mass estimate of LHS $1070 \mathrm{~A}$ is in good agreement with the empirical and theoretical predictions. We do not find any indication of a fourth component in the triple system LHS 1070 with mass down to $\sim 10 M_{\text {jup }}$. 


\section{Summary}

Using a combination of published and archival data and adding new observations from high spatial and spectral resolution imaging and spectroscopy, we have considerably improved the orbital solution for the low-mass triple system LHS 1070. Most noticeably we found that:

- The dynamical masses for LHS 1070 B and C, both close to the hydrogen burning limit, are in good agreement with theoretical predictions from the models of Baraffe et al. (1998) but higher by up to $25 \%$ than the empirical near infrared mass-luminosity relationship of Henry \& McCarthy (1993). Since only one datapoint (GJ $1245 \mathrm{C}$ ) is constraining the mass-luminosity function below $M_{V}=18$ (see Fig. 5), the addition of an accurate value for LHS 1070 BC marks an important anchorpoint for the mass-luminosity relation at the end of the main sequence.

- The orbits of the LHS 1070 BC system and the outer orbit of LHS 1070 A around the barycentre of LHS 1070 BC appear co-planar: their inclination angle agrees to within $2^{\circ}$ and the respective longitudes of the ascending node $(\Omega)$ of both orbits are identical within their uncertainties.

- The mass estimate of LHS 1070 A matches the predictions from theory and from the empirical tracks. Given its error margin and the fact that no companion could be identified in the well-resolved VLT/NACO images, we find no indication for a fourth component (LHS 1070 D) in close orbit around LHS $1070 \mathrm{~A}$.

Acknowledgements. We would like to thank the CRIRES science verification team for their work on CRIRES and for the execution of the observations.
We thank the anonymous referee for constructive comments. AS and TR acknowledge financial support from the Deutsche Forschungsgemeinschaft under DFG RE 1664/4-1 and DFG NE 515/23-1, respectively. AR acknowledges financial support through an Emmy Noether Fellowship from the Deutsche Forschungsgemeinschaft under DFG RE 1664/4-1.

\section{References}

Baraffe, I., Chabrier, G., Allard, F., \& Hauschildt, P. H. 1998, A\&A, 337, 403 Baraffe, I., Chabrier, G., Barman, T. S., Allard, F., \& Hauschildt, P. H. 2003, A\&A, 402, 701

Costa, E., Méndez, R. A., Jao, W.-C., et al. 2005, AJ, 130, 337

Delfosse, X., Forveille, T., Ségransan, D., Beuzit, J.-L., et al. 2000, A\&A, 364, 217

Diolaiti, E., Bendinelli, O., Bonaccini, D., Close, L. M., Currie, D. G., \& Parmeggiani, G. 2000, Proc. SPIE, 4007, 879

Donnison, J. R., \& Mikulskis, D. F. 1995, MNRAS, 272, 1

Harrington, R. S. 1975, AJ, 80, 1081

Henry, T. J., \& McCarthy, D. W., Jr. 1993, AJ, 106, 773

Henry, T. J., Franz, O. G., Wasserman, L. H., et al. 1999, ApJ, 512, 864

Hilditch, R. W. 2001, An Introduction to Close Binary Stars, ed. R. W. Hilditch (Cambridge, UK: Cambridge University Press), 392

Käufl, H. U., Amico, P., Ballester, P., et al. 2006, The Messenger, 126, 32

Kerber, F., Nave, G., Sansonetti, C. J., Bristow, P., \& Rosa, M. R. 2007, The Future of Photometric, Spectrophotometric and Polarimetric Standardization, ed. C. Sterken (San Francisco: ASP), ASP Conf. Ser., 364, 461

Leinert, C., Allard, F., Richichi, A., \& Hauschildt, P. H. 2000, A\&A, 353, 691

Leinert, C., Jahreiß, H., Woitas, J., et al. 2001, A\&A, 367, 183

Neuhäuser, R., Mugrauer, M., Seifahrt, A., Schmidt, T. O. B., \& Vogt, N. 2007, A\&A, 484, 281

Reiners, A., Seifahrt, A., Käufl, H. U., Siebenmorgen, R., \& Smette, A. 2007, A\&A, 471, L5

Shao, J., \& Dongsheng, T. 1996, Springer Series in Statistics: The Jackknife and Bootstrap 